\title{
Arbeit oder gemeinschaftliche Praxis? : Karl Marx im Spiegel neuerer Rezeptionstendenzen
}

In:

Deutsche Zeitschrift für Philosophie : Zweimonatsschrift der internationalen philosophischen Forschung. - ISSN: 2192-1482. - Berlin: Akademie Verlag

56 (2008) 3, S. 435-450 


\title{
LITERATURESSAY
}

\section{Arbeit oder gemeinschaftliche Praxis?}

\section{Karl Marx im Spiegel neverer Rezeptionstendenzen}

\author{
Von CHRISTINE WECKWERTH (Berlin)
}

Eine antike Auffassung besagt, dass es unmöglich ist, zweimal in denselben Fluss hineinzusteigen. „In dieselben Flüsse steigen wir und steigen wir nicht, wir sind und wir sind nicht." gilt übertragen auch für das Eintauchen in realisierte Theoriegebäude. Nachfolgende Generationen kehren unter gewandelten geschichtlichen Kontexten und Fragestellungen auf frühere Theorien zurück, die einst selbst im Flusse waren; es kommt zu Aspektverlagerungen, Umbildungen und auch Vereinseitigungen. Was bleibt, wie Hans Blumenberg einmal bemerkte, ist die Hypothek der Probleme, ,die auch und wieder zu wissen auferlegt, was schon einmal gewußt worden war". ${ }^{2}$ Den widersprüchlichen Fortbildungsprozess der nachhegelschen Philosophie hat exemplarisch Karl Löwith beschrieben. Rückwärts gesehen, konstatiert er bei den Junghegelianern und Marx einen kategorialen und methodischen Rückfall hinter Hegel, nach vorwärts erkennt er bei ihnen den produktiven Versuch, „die Fragestellungen der Philosophie gemäß dem faktisch veränderten Existenzbewußtsein dieser Generationen umzubilden". ${ }^{3}$ Als große Aufgabe der Vormärzgeneration hatte der Schriftsteller Heine schon früh die Emanzipation herausgestellt, die die Menschen und Völker vom Gängelbande der Bevorrechteten befreien sollte. ${ }^{4}$ Ihre Einlösung führte auf theoretischer Ebene zu einem Bruch mit der Spekulation Hegels, der auf unterschiedliche Weise vollzogen wurde, so vermittels kritischer Selbstbewusstseinsphilosophie, Anthropologie, Gesellschaftstheorie oder eines aufs einzelne Selbst zugeschnittenen Naturalismus. Der Marxsche Ansatz wurde durch seine Ausdehnung auf politische, ethische, erkenntnistheoretische und so weiter Gebiete selbst wieder transformiert - bis hin zu einer Kanonisierung als geschlossenes Weltanschauungssystem. Die Forderung, zum

1 Dieser Ausspruch Heraklits nach: Die Fragmente der Vorsokratiker, griech. u. dt. v. H. Diels, hg. v. W. Kranz, Bd. 1, 12. unveränd. Aufl., Zürich 1966, 22 B 49a.

2 H. Blumenberg, Die Legitimität der Neuzeit, erneuerte Ausg., 1. Aufl., Frankfurt/M. 1996, 59.

3 K. Löwith, Ludwig Feuerbach und der Ausgang der klassischen deutschen Philosophie, in: ders., Sämtliche Schriften, Bd. 5, Stuttgart 1988, 5.

4 H. Heine, Reisebilder. 3. Theil. Italien. 1828. I. Reise von München nach Genua, in: ders., Historisch-kritische Gesamtausgabe der Werke, in Verb. mit dem Heinrich-Heine-Institut hg. v. M. Windfuhr, Bd. 7/1: Reisebilder III/IV, bearb. v. A. Opitz, Düsseldorf 1986, 69. 
unverfälschten Ursprungskern einer Theorie zurückzukehren, unterschätzt das evolutionäre Moment, ebenso das kumulierte Potenzial geschichtlich erweiterter Problemstellungen und Antworten, selbst in deren Einseitigkeiten. Auch der Versuch einer „Destruktion des Überlieferungsgeschehens ${ }^{\text {“5 }}$ kann sich dem späteren zeitgeschichtlichen Horizont nicht entziehen.

Diese Überlegungen vorausgeschickt, wird sich Marx im Folgenden zunächst von der Seite der neueren Rezeptionsgeschichte angenähert. Dazu werden nacheinander die gegenwärtig einsetzende Rückbesinnung auf Marx' Kapitalismusanalyse, die für den angelsächsischen Sprachraum auszeichnende praktisch-ethische Marx-Rezeption sowie schließlich Habermas' Rekonstruktion des Historischen Materialismus betrachtet. ${ }^{6}$ Ein besonderes Augenmerk gilt der Frage nach dem Verhältnis von kritischer Strukturanalyse der Gesellschaft und Emanzipationskonzept, worauf diese Rezeptionslinien in unterschiedlicher Weise antworten. Es zeigt sich die Tendenz, dass beide von Marx zusammengedachten Theoriekomponenten darin voneinander abgetrennt werden. Um beide Seiten perspektivisch wieder zusammenzudenken, wird sich in einem zweiten Schritt dem Marxschen Ansatz zugewandt, der in erweiterter Form im Spiegel der sozialanthropologischen Wende Feuerbachs betrachtet wird. Dessen Wende zum Grundprinzip sozialer Relationalität wird hier als ein unausgeschöpftes theoriegeschichtliches Potenzial angesehen, das eine weitergehende Lösung hinsichtlich der aufgerollten Problematik birgt.

Blickt man auf die gegenwärtige Omnipotenz wirtschaftlicher Prozesse unter der Logik der Profitmaximierung, auf die Internationalisierung der Produktion und Finanzmärkte, die beschleunigte Entwicklung neuer Technik und Technologien bei einhergehender Krisenanfälligkeit der Kapitalbewegungen, auf die Wirksamkeit des Effizienzprinzips bis in die individuellen Biographien hinein, auf eine wachsende Ungleichheit wie den unfreiwilligen Ausschluss vieler Bürger aus der Arbeitswelt, scheint die empirische Wirklichkeit Marx' kritischer Gesellschaftsanalyse regelrecht entgegenzuarbeiten, wie im gleichen Zuge seine Frage nach Transformation dieser Sozialitätsstufe auf die Tagesordnung gesetzt wird. Die gegenwärtige Entwicklung hat augenscheinlich ein erneutes Interesse an Marx hervorgerufen, das bis in die Feuilletons vorgedrungen ist. ${ }^{7}$ Man spricht inzwischen wieder von einer Renaissance seiner Theorie, deren Rezeption in einem Umbruchsprozess gesehen wird. ${ }^{8}$ Es wird zugleich die Forderung laut, das Kapital neu zu lesen ${ }^{9}$ - Marx wird im Land seiner Herkunft allmählich

5 Von einer „Destruktion des Überlieferungsgeschehens“ spricht Christoph Henning, der damit das originäre Marxsche Denken neubeleben und es zugleich von der ,,Verfallsgeschichte“ der marxistischen und auf Marx reagierenden Theorie" befreien will; ders., Philosophie nach Marx. 100 Jahre Marxrezeption und die normative Sozialphilosophie der Gegenwart in der Kritik, Bielefeld 2005, 23, 24; vgl. dazu auch die Rezension von I. Fetscher, Was Philosophen von Marx verstanden haben, in: Marx-Engels-Jahrbuch 2006, hg. v. der Internationalen Marx-Engels-Stiftung, Berlin 2007, 257-263.

6 Es wird hierbei keine ausgewogene Darstellung der neueren Marx-Rezeption oder gar Vollständigkeit angestrebt; der Rückgriff auf diese Rezeptionstendenzen erfolgt vielmehr aus einer bestimmten problemgeschichtlichen Sicht.

7 Man vergleiche die Vielzahl an Zeitungsartikeln, die anlässlich von Marx' 125. Todestag um den 14. März 2008 erschienen sind.

8 Vgl. J. Rohbeck, Marx (Grundwissen Philosophie), Leipzig 2006, 7. Zu einem vertiefenden Verständnis der Marxschen Theorie trägt zugleich die historisch-kritische Gesamtausgabe der Schriften von Marx und Engels (MEGA²) bei, deren 2. Abteilung „,Das Kapital“ und Vorarbeiten“ bald vollständig vorliegen wird.

9 Vgl. J. Hoff u. a. (Hg.), Das Kapital neu lesen - Beiträge zur radikalen Philosophie, Münster 2006; oder bereits: Marx's method in Capital: a reexamination, hg. v. F. Moseley, Atlantic Highlands/NJ 
wieder eingebürgert. ${ }^{10}$ Eine Chance für eine neue, kritische Rezeption wird unter anderem darin gesehen, dass nach dem Untergang des Realsozialismus als der scheinhaften Leibwerdung der marxistischen Theorie nunmehr eine Marx-Lektüre jenseits verzerrender Ideologien und parteiischer Interessen stattfinden kann. Verschiedene Autoren streben in diesem Kontext eine Rückbesinnung auf Marx an, die sich bewusst vom traditionellen Marxismus abgrenzt, bis hin zur Forderung, die Wirkungsgeschichte der Marxschen Theorie überhaupt zu negieren. ${ }^{11}$

Was den deutschsprachigen Raum betrifft, sind in den letzten Jahren in diesem Zusammenhang verschiedene auf Marx' ökonomisch begründete Gesellschaftstheorie zurückgehende Bücher erschienen. ${ }^{12}$ Marx ersteht darin vor allem als kritischer Kapitalismusanalytiker wieder, der die moderne Gesellschaft auf ökonomische Bewegungsgesetze zurückführt. Ins theoretische Blickfeld tritt damit die Vergegenständlichungsproblematik, wie sie sich auf der ökonomischen Ebene von der Arbeit kooperierender Individuen in der Doppelfunktion einer Tausch- und Gebrauchswert erzeugenden Größe zur versachlichten, widersprüchlichen Bewegung des sich selbst verwertenden Kapitals erstreckt. Marx zufolge bestimmt dieser Vermittlungsprozess in fundamentaler Weise den sozialen, politischen und geistigen Lebensprozess. Hinter der Neubesinnung auf seine kritische Gesellschaftsanalyse steht zu Recht die Intention, der im Ganzen spontan ablaufenden gegenständlich-praktischen Objektivierungssphäre innerhalb der Gesellschaftstheorie beziehungsweise Sozialphilosophie wieder einen systematischen Stellenwert einzuräumen. ${ }^{13}$ Mit dem Rekurs auf Marx' politökonomische Theorie wird zum einen die Aufgabe hervorgerufen, diese Theorie, die Marx selbst fragmentarisch hinterlassen hat, auch im Hinblick auf die nicht behandelten Sachgebiete weiter auszubauen sowie sie mit Erscheinungen des gegenwärtigen globalisierten Kapitalismus zu vermitteln - eine Aufgabe, die zwar gestellt, jedoch nicht gelöst ist. Man wirft zum anderen die Frage auf, inwieweit sich unter den Bedingungen verselbständigter gesellschaftlicher Strukturverhältnisse die Dimension von Selbstbestimmung, universaler Fähigkeitenentwicklung oder auch eines solidarischen Umgangs der Menschen überhaupt denken lässt. Letztere Fragestellung tritt in den betrachteten Arbeiten nach Auffassung der Verfasserin mehr oder weniger zurück. Eine solche Hintanstellung findet

1993. Der Kreis um Fred Moseley hat nachfolgend verschiedene Sammelbände zu einzelnen Bänden des Marxschen Kapitals herausgegeben.

10 Axel Honneth hat 2002 dagegen noch von „Tendenzen einer geistigen Ausbürgerung von Marx aus Deutschland“" gesprochen, wodurch nach ihm eine paradoxe Ungleichzeitigkeit zwischen Deutschland und den anderen Ländern des Westens entstanden ist; vgl. ders., Schwerpunkt: Marx in der Diskussion, in: Deutsche Zeitschrift für Philosophie, 50 (2002) 3, 393; ebenfalls ders., Philosophie. Eine Kolumne. Ungleichzeitigkeiten der Marx-Rezeption, in: Merkur, Heft 603, 53. Jg., 1999, 643-651.

11 Für eine Unterscheidung zwischen Marx und dem traditionellen Marxismus plädiert etwa Christian Iber. Der Marxismus in all seinen Spielarten hat nach ihm die Komplexität der Marxschen Theorie reduziert, die es wieder zu vergegenwärtigen gilt. Vgl. ders., Grundzüge der Marx'schen Kapitalismustheorie, Berlin 2005, 11, 18; zur Sichtweise auf Marx ohne Marxismus vgl. die einleitenden Ausführungen.

12 Neben den bereits angeführten Arbeiten vgl. stellvertretend M. Berger, Karl Marx: „Das Kapital“. Eine Einführung, München 2003; M. Iorio, Karl Marx. Geschichte, Gesellschaft, Politik. Eine Einund Weiterführung, Berlin 2003; M. Heinrich, Kritik der politischen Ökonomie. Eine Einführung, Stuttgart 2004; ders., Wie das Marxsche Kapital lesen? Hinweise zur Lektüre und Kommentar zum Anfang von „Das Kapital“, Stuttgart 2006.

13 Diese Intention richtet sich insbesondere gegen eine konstatierte Vorherrschaft rationaler und formaler Argumentationen im sozialphilosophischen Diskurs; vgl. Ch. Henning, Philosophie nach Marx ..., a. a. O., bes. 25 f., $564 \mathrm{ff}$. 
sich beispielsweise in der Deutung von Marx' Kapitalismustheorie als einem ,,radikal negativistischen" Ansatz ${ }^{14}$, mit dem die Frage nach der geschichtlichen Perspektive nicht mehr darzulegen ist. Oder die Emanzipationsthematik wird entschärft, etwa wenn in einer MarxStudie darauf verwiesen wird, dass nicht mehr die Frage von Entfremdung oder Aufhebung zur Debatte steht, sondern es darauf ankommt, ,mit Fremdheitserfahrungen möglichst vernünftig umzugehen“" und ,,in der von uns als fremd erfahrenen Welt sinnvoll zu leben“. ${ }^{15}$ Angesichts der vom Autor herausgestellten Dominanz der Kapitalverwertungsprozesse ist zu fragen, wie sich in einer verkehrten Welt de facto vernünftig (politisch) einzurichten ist. Mit dieser Wendung wird die Marxsche Programmatik offensichtlich beschnitten; ging es dem Denker im Exil doch gerade darum, kritische Gesellschaftstheorie und Emanzipationsfrage zusammenzudenken. ${ }^{16}$ Diese Programmatik entsprang dem Bestreben, zwei Grundtendenzen der europäischen Kulturgeschichte zu verbinden - die neuzeitliche Traditionslinie der rational-operationalen Selbstverständigung des Subjekts mit der plebejischen Traditionslinie solidarischer Gleichheit. ${ }^{17}$

Gegenüber einer solchen Wiederbelebung von Marx' politökonomisch fundierter Gesellschaftstheorie stößt man insbesondere im angelsächsischen Raum auf die Tendenz, Marx im Lichte praktisch-ethischer Fragestellungen zu reflektieren. ${ }^{18}$ Mit dieser Ausrichtung gewinnt zugleich die Emanzipationsthematik an Bedeutung. Die angelsächsische Marx-Diskussion ist auf diese Tendenz allerdings nicht zu beschränken. ${ }^{19}$ Zur Vergegenwärtigung der praktischethischen Rezeption wird im Folgenden auf einen repräsentativen Text von Alasdair MacIn-

14 Vgl. Ch. Iber, Grundzüge der Marx'schen Kapitalismustheorie, a. a. O., bes. 16-18.

15 J. Rohbeck, Marx (Grundwissen Philosophie), a. a. O., 66. Ungeachtet der angeführten Tendenz gibt Rohbecks einführende Studie einen sachlichen, ausgewogenen Überblick über die Marxsche Theorie im Kontext des gesamten Werkes.

$16 \mathrm{Zu}$ dieser Problematik vgl. A. Honneth, Logik der Emanzipation. Zum philosophischen Erbe des Marxismus, in: H. L. Krämer u. C. Leggewie (Hg.), Wege ins Reich der Freiheit. André Gorz zum 65. Geburtstag, Berlin 1989, 94.

17 Vgl. dazu G. Irrlitz, Notizen zu Marx, nach dem Marxismus, in: Marxismus. Versuch einer Bilanz, Magdeburg 2001, bes. $71 \mathrm{ff}$.

18 Anzeichen einer Marx-Renaissance im englischsprachigen Raum hat Axel Honneth bereits vor längerer Zeit konstatiert, wobei er sich vor allem auf die praktisch-ethische Rezeption bezogen hat. Der Marxsche Ansatz wird nach ihm hier tendenziell als Entwurf einer politischen Ethik rezipiert, und zwar im Rahmen einer Selbstkritik des Liberalismus. Vgl. ders., Philosophie. Eine Kolumne. Ungleichzeitigkeiten der Marx-Rezeption, a. a. O., 644; dazu stellvertretend A. MacIntyre, The Theses on Feuerbach: a road not taken, in: Artifacts, representations and social practice: essays for Marx Wartofsky, hg. v. C. C. Gould u. R. Cohen, Dordrecht 1994, 277-290; dt.: Marx’ „Thesen über Feuerbach“ - ein Weg, der nicht beschritten wurde, in: Deutsche Zeitschrift für Philosophie, 44 (1996) 4, 543-554; R. Geuss, Freiheit im Liberalismus und bei Marx, in: J. Nida-Rümelin u. W. Vossenkuhl (Hg.), Ethische und politische Freiheit, Berlin 1998, 114-125; D. Brudney, Marx's attempt to leave philosophy, Cambridge 1998; R. T. Sweet, Marx, morality and the virtue of beneficence, Lanham 2002; M. C. Nussbaum, Langfristige Fürsorge und soziale Gerechtigkeit. Eine Herausforderung der konventionellen Ideen des Gesellschaftsvertrages, in: Deutsche Zeitschrift für Philosophie, 51 (2003) 2, 179-198.

19 Vgl. den schon länger vorliegenden Literaturbericht von M. Quante, Zeit für Marx? Neuere Literatur zur Philosophie von Karl Marx, in: Zeitschrift für philosophische Forschung, Bd. 56, Frankfurt/M. 2002, 450-467. Bezogen vor allem auf den angelsächsischen Sprachraum stellt Quante darin drei thematische Schwerpunkte heraus: die Hegel-Marx-Connection, den Materialismus und die Kritik der politischen Ökonomie, eingeschlossen Marx' Verhältnis zu einer ökologischen Ethik. Vgl. auch R. D. Wolff, Die überdeterministische und klassentheoretische Kapital-Lektüre in den USA, in: Das Kapital neu lesen ..., a. a. O., 128-145. 
tyre zurückgegriffen, in dem eine Neuinterpretation der Marxschen „Feuerbach-Thesen“ unternommen wird. MacIntyre geht auf diesen frühen Programmtext von Marx zurück, um davon einen Weg zu beschreiten, den dieser selbst nicht gegangen ist, dem Autor zufolge aber hätte gehen können (554). ${ }^{20}$ Den Angelpunkt seiner Interpretation bildet die Umdeutung des von Marx gebrauchten Begriffs der ,gegenständlichen Tätigkeit“, den er in Richtung eines Praxistyps deutet, der teleologisch auf ein gemeinsam Gutes geordnet ist und bei dem die kooperierenden Individuen zugleich ihre eigenen Zwecke erreichen. MacIntyre greift dazu auf die aristotelische Tradition zurück, wobei er eine Abweichung von Marx' ursprünglicher Bestimmung bewusst in Kauf nimmt. Die Teilnahme an der gemeinsamen Praxis bewirkt bei den Einzelnen eine „Umwandlung der ursprünglich in die Tätigkeit mit eingebrachten Wünsche“ (546). Durch eine solche Umwandlung ihrer Wünsche wie die Aneignung derjenigen intellektuellen und moralischen Tugenden wie derjenigen intellektuellen, körperlichen und imaginativen Fähigkeiten, die zum Erreichen der Güter der gemeinsamen Praxis notwendig sind, verwandeln sich die Individuen weitergehend selbst (546).

MacIntyre spielt hier auf die dritte „Feuerbach-These“ an, die besagt, dass das Ändern der Umstände und die menschliche Tätigkeit oder auch Selbstveränderung zusammenfallen, was Marx auch als „revolutionaire Praxis“ bezeichnet hat. ${ }^{21}$ Beispiele für den gemeinschaftlichen Praxistyps sind nach MacIntyre das Gemeinschaftsleben der Handwerker am Ende des 18. und Beginn des 19. Jahrhunderts, Fischercrews, Landkooperativen oder auch Streichquartette (551 f., 554). Die Menschen verwirklichen hier Vorstellungen eines gemeinsamen Gutes und von Tugenden, die bereits jenseits einer individualistischen Praxis liegen. Der Autor erkennt darin das entscheidende Potenzial zum Widerstand gegen diese Sozialitätsform; nur, ,in solchen Praktiken und durch sie kann über den Standpunkt der bürgerlichen Gesellschaft hinausgegangen werden“ (554). Die gesellschaftliche Transformation der bürgerlichen Gesellschaft wird von ihm entsprechend an eine moralische Änderung der interagierenden Individuen gebunden, womit er zu Prämissen gelangt, die dem Marxschen Ansatz augenscheinlich widersprechen. Stimmt seine Interpretation mit Marx darin überein, wonach die individualistische bürgerliche Gesellschaft nicht durch Theorie, sondern nur praktisch zu überschreiten ist $(545)^{22}$, ebenfalls in dem Gedanken, individuelle Selbstverwirklichung nur im Horizont gemeinschaftlicher Praxis zu fassen - „erst in der Gemeinschaft existiren für jedes Individuum die Mittel, seine Anlagen nach allen Seiten hin auszubilden; erst in der Gemeinschaft wird also die persönliche Freiheit möglich“"23, wie es bei Marx heißt -,

20 Die folgenden in Klammern stehenden Seitenangaben beziehen sich auf die deutsche Fassung des Textes; vgl. A. MacIntyre, Marx’ „Thesen über Feuerbach“ „.., a. a. O.

21 K. Marx, Notizbuch aus den Jahren 1844-1847. 1) ad Feuerbach., in: K. Marx u. F. Engels, Gesamtausgabe, hg. v. der Internationalen Marx-Engels-Stiftung (im Folgenden: MEGA²), Berlin 1998, Bd. $\mathrm{IV} / 3,20$.

22 Eine Theorie, die die bürgerliche Gesellschaft allein in Begriffen von Nützlichkeit, Vertrag und individuellen Rechten denkt, gehört nach MacIntyre selbst noch diesem besonderen Typ gesellschaftlicher Ordnung an (544). Auch Gesellschaftstheoretikern wie Parson, Sartre, Habermas oder Bourdieu wirft MacIntyre vor, dass ihre Lösungen im Hinblick auf das Problem, den Menschen als Produkt objektiver gesellschaftlicher und natürlicher Umstände sowie als autonom (rational) Handelnden zu begreifen, innertheoretisch beziehungsweise an der wissenschaftlichen Praxis orientiert bleiben (550). Marx kam es nach ihm gerade darauf an, „daß es aus dem Inneren der Theorie oder selbst aus dem Inneren der Praxis des Theoretikers keine Lösung geben kann" (550).

23 Zitiert nach der Vorabpublikation von Textzeugen der Deutschen Ideologie: K. Marx, F. Engels, J. Weydemeyer, Die Deutsche Ideologie. Artikel, Druckvorlagen, Entwürfe, Reinschriftfragmente und Notizen zu I. Feuerbach und II. Sankt Bruno. Text (im Folgenden: K. Marx u. F. Engels, Die 
so weicht sie in einer grundlegenden Frage vom Marxschen Konzept ab. MacIntyre geht es um eine auf Tugenden ausgerichtete Praxis in lokalen Gemeinschaften ${ }^{24}$, die er nicht in der Marxschen Perspektive auf ihre objektiven strukturellen Voraussetzungen hin reflektiert. Er setzt die gemeinsam zu erreichenden Güter und Zwecke als feste gesellschaftliche Ordnungsgrößen voraus, ohne sie nach ihrem Ursprung im Bezugsfeld menschlicher Tätigkeit zu hinterfragen. Wie sich die gemeinsamen Zwecke und Güter im Rahmen der individualistischen bürgerlichen Gesellschaft konstituieren und behaupten beziehungsweise sich lokale Gegenseitigkeit und partiale Solidarität überhaupt gegen die globalen Gesetze der Kapitalverwertung und Konkurrenz wie die bürokratischen Staatsapparate durchsetzen können, bleibt in MacIntyres Interpretation offen. Er trennt die Transformations- allgemein von der Strukturproblematik der bürgerlichen Gesellschaft ab, zu der er durch Festschreibung des Marxschen Tätigkeitsbegriffs auf die moralische Praxis keinen Zugang mehr hat. Der Kommunitarist tritt damit sozusagen als Pendant zur gegenwärtigen deutschen Marx-Diskussion auf.

Den Weg einer Vermittlung von ökonomischer und moralisch-sozialintegrativer Evolution hat unter anderen Jürgen Habermas in seiner Rekonstruktion des historischen Materialismus eingeschlagen. ${ }^{25}$ Sein Rekonstruktionsversuch fällt in eine frühere Rezeptionswelle der Marxschen Theorie, die im Zuge der 68er-Bewegung einsetzte. Neben dem Bestreben, die geschichtsmaterialistische Basistheorie einer Revision zu unterwerfen und auf weitere soziokulturelle Regionen und geschichtliche Epochen auszudehnen, war ein zentrales Motiv dieser theoretischen Bewegung, ausgehend von Prämissen des historischen Materialismus, die Emanzipationsproblematik neu zu durchdenken. Die klassischen Grundbestandteile des historischen Materialismus sollten so umformuliert werden, dass ,,sie den politischen Aktionszusammenhang aktueller Emanzipationsbewegungen verständlich machen“. ${ }^{26}$ Dieser Option lag die Einsicht zu Grunde, dass die Marxsche Revolutionstheorie für die Zeit des Spätkapitalismus kein adäquates Transformationsmodell mehr bereitstellt. Die Emanzipationsfrage avancierte vor diesem Hintergrund zu einem treibenden Motiv dieser Bewegung. ${ }^{27}$ Gegenüber den heute vorherrschenden Theoriedebatten sah man im Marxschen Ansatz damals ein durchaus anschlussfähiges Theoriepotenzial, so auch Habermas. Im Unterschied zu strukturalistischen Deutungen erkennt er im Marxschen Ansatz vornehmlich eine Theorie der sozialen Evolution $(9,41)$. Gegenüber MacIntyre zeichnet seine Interpretation aus, Marx’ Begriff der gegenständ-

deutsche Ideologie, Vorabdruck), in: Marx-Engels-Jahrbuch 2003, hg. v. der Internationalen MarxEngels-Stiftung, Berlin 2004, 73; ebenfalls K. Marx u. F. Engels, Die deutsche Ideologie. Kritik der neuesten deutschen Philosophie in ihren Repräsentanten Feuerbach, B. Bauer und Stirner, und des deutschen Sozialismus in seinen verschiedenen Propheten, in: dies., Werke (im Folgenden: MEW), Bd. 3, Berlin 1958, 74.

24 Vgl. in diesem Zusammenhang auch A. MacIntyre, After virtue: a study in moral theory, London 1981; dt.: Der Verlust der Tugend. Zur moralischen Krise der Gegenwart, Frankfurt/M. 1995.

25 Vgl. J. Habermas, Zur Rekonstruktion des Historischen Materialismus, 3. Aufl., Frankfurt/M. 1982. Folgende Ausführungen gehen vornehmlich auf diesen Sammelband zurück, auf den sich ebenfalls die folgenden in Klammern stehenden Seitenangaben beziehen.

26 A. Honneth u. U. Jaeggi (Hg.), Theorien des Historischen Materialismus, Frankfurt/M. 1977, 18.

27 Man stößt in diesem Zusammenhang auf praxisphilosophische, strukturalistische, kommunikationstheoretische, später auch auf spiel-, kultur- oder machtheoretische Neuinterpretationen der Marxschen Theorie; vgl. A. Honneth u. U. Jaeggi (Hg.), Theorien des Historischen Materialismus, a. a. O.; und dies. (Hg.), Arbeit, Handlung, Normativität. Theorien des Historischen Materialismus 2, Frankfurt/M. 1980; zu den letzten drei Interpretationsrichtungen vgl. A. Honneth, Logik der Emanzipation. Zum philosophischen Erbe des Marxismus, a. a. O., 87-94. 
lichen Tätigkeit oder auch Praxis spezifisch als Arbeit zu deuten, die er bei Marx zur fundamentalen Syntheseform der Gesellschaft aufsteigen sieht. ${ }^{28}$

Habermas versteht darunter ein zweckrationales (instrumentelles, strategisches) Handeln, das eine zielgerichtete Umformung von Material nach technischen Regeln sowie ein am eigenen Erfolg orientiertes Handeln umschreibt $(145,32)$. Er rechnet diesem Handlungstypus das objektive Bezugssystem der Produktivkräfte zu, an dessen Entwicklung Marx den Grundprozess der sozialen Evolution festgemacht hat (11). Erkennt Habermas dem systematischen Rückgang auf die Arbeit bezogen auf die Evolutionstheorie einen reellen Gehalt zu, insofern Marx hier auf einen unaufhebbaren Faktor gesellschaftlicher Reproduktion zurückgeht, so stellt dieser Ansatz nach ihm gleichwohl eine zu schmale Basis dar, den menschlichen Evolutionsprozess zu begründen; kommt der Entwicklungslogik der sozialen Integration, im Kernbereich Moral und Recht, darin doch kein Eigengewicht zu beziehungsweise wird diese unzulässig auf den zweckrationalen Handlungstyp reduziert. ${ }^{29}$ Habermas ergänzt den Marxschen Ansatz in der Folge um einen zweiten Handlungstypus, das kommunikative Handeln, das ein rational motiviertes, auf Verständigung und Einigung ausgehendes Handeln darstellt, bei dem intersubjektiv geltende Normen befolgt werden. Es steht für einen reziproken Prozess zwischen Personen, dem die Erfahrung ,der zwanglos einigenden, konsensstiftenden Kraft argumentativer Rede“ zu Grunde liegt. Die Teilnehmer überwinden hier ihre zunächst nur subjektiven Auffassungen und vergewissern sich dank der Gemeinsamkeit vernünftig motivierter Überzeugungen gleichzeitig der Einheit der objektiven Welt und der Intersubjektivität ihres Lebenszusammenhanges. ${ }^{30}$ Diesem Handlungstypus ordnet Habermas die Seite der Produktionsverhältnisse zu, die nach ihm Ausdruck der Formen des sozialen Verkehrs beziehungsweise der sozialen Integration sind. Eine für die Neufassung des historischen Materialismus zentrale These lautet, dass der „Schrittmacher der sozialen Evolution“ die Entwicklung der normativen Strukturen ist, wie sie sich in den Vermittlungen kommunikativen Handelns, den Konfliktregelungsmechanismen, den Weltbildern und Identitätsformationen verkörpern (35). Orientiert an Entwicklungstheorien bei Mead, Piaget, Freud oder Chomsky, knüpft Habermas diese Entwicklung an kognitive Lernprozesse auf individueller und gesellschaftlicher Ebene. Evolutionäre Lernprozesse vollziehen sich Habermas zufolge in Gestalt einer institutionellen Verkörperung von Rationalitätsstrukturen (37). Die soziokulturelle Evolution folgt so in letzter Instanz der Logik argumentativer Rede.

In seiner Ergänzung des historischen Materialismus um den Typus des kommunikativen Handelns öffnet Habermas die Struktur- und Evolutionstheorie der Gesellschaft einer Soziali-

28 Die Arbeit tritt bei Marx nach Habermas an die Stelle, die vormals dem idealistischen Bewusstseinsbegriff zugekommen ist; vgl. dazu J. Habermas, Marxens Metakritik an Hegel: Synthesis durch gesellschaftliche Arbeit, in: ders., Erkenntnis und Interesse. Mit einem neuen Nachwort, 9. Aufl., Frankfurt/M. 1988, 36-59.

29 Marx hat nach Habermas versucht, ,„den welthistorischen Bildungsprozeß der Menschengattung aus Gesetzen der Reproduktion des gesellschaftlichen Lebens zu rekonstruieren. Den Mechanismus der Veränderung des Systems gesellschaftlicher Arbeit findet er im Widerspruch der durch die Arbeit akkumulierten Verfügungsgewalt über Naturprozesse und dem institutionellen Rahmen naturwüchsig geregelter Interaktionen. Allein, die genaue Analyse des ersten Teils der Deutschen Ideologie zeigt, daß Marx nicht eigentlich den Zusammenhang von Interaktion und Arbeit expliziert, sondern unter dem unspezifischen Titel der gesellschaftlichen Praxis eins auf das andere reduziert, nämlich kommunikatives Handeln auf instrumentales zurückführt.“ (J. Habermas, Arbeit und Interaktion. Bemerkungen zu Hegels Jenenser „Philosophie des Geistes“, in: ders., Technik und Wissenschaft als „Ideologie“, 9. Aufl., Frankfurt/M. 1978, 45)

30 Vgl. J. Habermas, Theorie des kommunikativen Handelns, Bd. 1, Frankfurt/M. 1988, 28. 
sierungskomponente, die bei Marx eine stark eingeschränkte Darstellung gefunden hat - Marx reflektiert diese allein bezogen auf die zukünftige kommunistische Gesellschaft sowie partiell auf die Arbeiterbewegung und ihre Organisation. ${ }^{31}$ Die berechtigte Ergänzung des historischen Materialismus führt bei Habermas zu einem evolutionstheoretischen Konzept, worin der soziale Progress allein den moralisch-kommunikativen Lernprozessen überantwortet wird. Die Sphäre der materiellen Vergegenständlichung besitzt dagegen einen untergeordneten Stellenwert, und zwar im Sinne von ,evolutionären Herausforderungen ungelöster, ökonomisch bedingter Systemprobleme“ (12). Die Lösung dieser Probleme obliegt allein rationalen moralischen Lernprozessen. Auch hier wird in der Folge das tradierte Basis-Überbau-Schema umgekehrt. Die methodische Unterscheidung zweier Handlungstypen führt bei Habermas zur Annahme zweier separater Vergesellschaftungsprozesse: die materielle Reproduktion mit ihren Subsystemen Markt (Geld) und Staat (Macht) sowie die symbolische Reproduktion, die in der auf Konsens gehenden kommunikativen Alltagspraxis einsetzt. Beide konstituieren zwei relativ selbständige Wirklichkeitssphären mit eigener Raum- und Zeitstruktur, womit Marx' durchgehendes Schichtenmodell der gesellschaftlichen Verhältnisse aufgelöst wird. An die Stelle einer einheitlichen Theorie des zivilisatorischen Prozesses treten nebeneinander eine empirische Gesellschaftstheorie sowie eine logisch begründete Handlungstheorie sprachlich-rationaler Verständigungs- und Willensbildungsakte. Der kommunikationstheoretische Ansatz richtet sich bezeichnenderweise nicht auf den empirischen Prozess; es geht hier vielmehr darum, ,daß strukturelle Möglichkeiten der Rationalisierung des Handelns eröffnet werden“" (37), das heißt um die abgewandelte Kantsche Frage nach den Bedingungen der Möglichkeit rationalen Handelns. Wie die kognitiven Lernpotenziale mit den verselbständigten Strukturen der Systemwelt zusammengeschlossen werden, um soziale Evolutionsprozesse in Gang zu bringen, bleibt damit unterbestimmt.

Ebenfalls Habermas' intersubjektive Wendung des historischen Materialismus führt auf das Problem, ökonomische Struktur- und soziale Transformationstheorie zusammenzudenken, vorausgesetzt man will bei der aufgezeigten Kluft nicht stehen bleiben. Eine weiterführende Antwort darauf liegt, wie ich denke, weder in einer bloßen Restitution der Marxschen Theorie noch in einer idealen Ergänzung oder praktisch-ethischen Umdeutung dieser Theorie; sie liegt, wenn überhaupt, in Richtung einer immanenten Erweiterung der anthropologischen Basis und der entsprechenden phänomenologischen Objektivierungstheorie. Dazu kann man auf spätere anthropologische, soziologische, ökonomische oder gesellschaftstheoretische Ansätze zurückgreifen. Der Weg, der hier eingeschlagen werden soll, führt hinter Marx wieder zurück. Eine erweiterte Basis lässt sich meines Erachtens aus Feuerbachs sozialanthropologischer Wende der Subjektphilosophie beziehen, deren Boden die Marxsche Theorie eigens entwachsen ist. ${ }^{32}$ Diese Wende resultierte bei Feuerbach aus einem Zusammenführen der rationalen Subjekttheorie des deutschen Idealismus mit pantheistischen und gefühlsphilosophischen Ansätzen, so bei Bruno, Spinoza, Herder, Jacobi oder Schleiermacher. Als seine theoretische Leistung hat sein Nachfolger Marx herausgestellt, dass er den wahren Materi-

31 Ein bewusster freier Verkehr der Menschen ist für Marx erst Resultat der menschlichen Gattungsgeschichte beziehungsweise nur auf ein exklusives Subjekt - die Arbeiterklasse - zu beziehen. Bezogen auf den Kommunismus antizipiert er einen Zustand, in dem sich solidarisch verbundene, gleiche Individuen bewusst vereinigen, um die erzeugten Produktions- und Verkehrsverhältnisse unter ihre Kontrolle und Macht zu bringen. Vgl. K. Marx u. F. Engels, Die deutsche Ideologie, Vorabdruck, $73 \mathrm{f}$. u. 22, 87 ff. (MEW, Bd. 3, 74, 35, 66 ff.).

32 Vgl. dazu etwa H. Joas, Thesen über Feuerbach und Marx: Intersubjektivität und historischer Materialismus, in: A. Leist (Hg.), Ansätze zur materialistischen Sprachtheorie, Kronberg/Ts. 1975, $46-54$. 
alismus begründet hat, der „das gesellschaftliche Verhältniß das ,des Menschen zum Menschen" - ebenso zum Grundprincip der Theorie macht" ". ${ }^{33}$ Feuerbachs entscheidender Schritt bestand im Anschluss an den klassischen Idealismus darin, an die Stelle des substanzial gefassten Subjektbegriffs als der übergreifenden Identität von Subjekt und Objekt einen Verhältnisbegriff zu setzen, den er spezifisch von zwischenmenschlichen Beziehungen her dachte. An Hegels genetischem Phänomenologiekonzept hielt Feuerbach grundlegend fest. Das höchste und letzte Prinzip der Philosophie, wie es in seinen Grundsätzen der Philosophie der Zukunft lautet, „,ist daher die Einheit des Menschen mit dem Menschen. Alle wesentlichen Verhältnisse - die Prinzipien verschiedener Wissenschaften - sind nur verschiedene Arten und Weisen dieser Einheit. "“34 Diese Einheit stützt sich nach ihm in elementarer Form auf die „Realität des Unterschieds von Ich und $\mathrm{Du}^{\text {“35 }}$, welchen Bezug er im Spannungsfeld leiblicher, bedürfnishaft-volitiver, emotionaler, phantastisch-fiktiver und sprachlich-logischer Akte dachte. ${ }^{36}$ Feuerbach selbst hat diese Programmatik nur fragmentarisch, vor allem auf religionsphilosophischem Gebiet, realisiert. Sein Nachfolger Marx hat das Unfertige seiner Philosophie bald erkannt und das zwischenmenschliche Verhältnis bereits in seinen Ökonomisch-Philosophischen Manuskripten als ein gesellschaftliches gedeutet. ${ }^{37}$ Ins Zentrum treten bei ihm die geschichtlich realisierten Produktionen interagierender Individuen, auf deren Grundlage sich ein objektives Gefüge gesellschaftlicher Verhältnisse aufspannt, das unabhängig von deren Willen besteht und diese unhintergehbar bestimmt. Im Gegenzug zum Idealismus Hegels wie der Junghegelianer begründet er das Gefüge der gesellschaftlichen Verhältnisse auf der Produktion des materiellen Lebens. ${ }^{38}$ Die gegenseitigen Ich-Du-Bezüge erhalten in der Folge den Status eines abgeleiteten Verhältnisses, womit Feuerbachs sozialanthropologische Basis überformt und zugleich eingeschränkt wird. Bereits im Umfeld ihres Projektes Deutsche Ideologie sehen Marx und Engels in der „Einheit von Ich und Du“ nur eine mysteriöse Form, hinter der sie allein eine natürliche Beziehung - den Geschlechtsakt

33 K. Marx, Ökonomisch-philosophische Manuskripte (Zweite Wiedergabe), in: MEGA², Bd. I/2, 400. In dem Verhältnis des Menschen zum Menschen, in seiner natürlichen Form zwischen Mann und Frau, erkennt der junge Marx den Maßstab der geschichtlichen Vergesellschaftung des Menschen. Aus diesem Verhältnis „,kann man also die ganze Bildungsstufe d[es] Menschen beurtheilen“. Darin zeigt sich zugleich, ,inwieweit das Bedürfniß des Menschen zum menschlichen Bedürfniß, inwieweit ihm also der andre Mensch als Mensch zum Bedürfniß geworden ist, inwieweit er in seinem individuellsten Dasein zugleich Gemeinwesen ist" (ebd., 388). In diesen und anderen Passagen erweist sich der junge Marx ungeteilt als Feuerbachianer.

34 L. Feuerbach, Grundsätze der Philosophie der Zukunft, in: ders., Gesammelte Werke (im Folgenden: FGW), hg. v. W. Schuffenhauer, Bd. 9, 2., durchges. Aufl., Berlin 1982, 340.

35 Ebd., 339; vgl. auch L. Feuerbach, Über Spiritualismus und Materialismus, besonders in Beziehung auf die Willensfreiheit, in: FGW, Bd. 11, 171: „Das wirkliche Ich ist nur das Ich, dem ein Du gegenübersteht und das selbst einem andern Ich gegenüber Du, Objekt ist.“

36 Vgl. dazu Ch. Weckwerth, Ludwig Feuerbach zur Einführung, Hamburg 2002, bes. 81-100.

37 Vgl. auch Marx' Brief an Feuerbach vom 11. August 1844, in: $\mathrm{MEGA}^{2}$, Bd. III/1, 63.

38 Diese Programmatik entwickelt Marx gemeinsam mit Engels zum ersten Mal systematisch in der zu ihren Lebzeiten nicht erschienenen Deutschen Ideologie. Neben der Produktion des materiellen Lebens und der Erzeugung neuer Bedürfnisse führen sie in dieser Schrift als humanspezifische Voraussetzungen ebenfalls die Produktion des menschlichen Lebens im Rahmen der Familie (Zeugung) und die körperliche Organisation der Menschen wie das daraus resultierende Verhältnis zur Natur an, als eine bereits abgeleitete Form auch die Sprache. Den eigentlichen Unterschied zum Tier setzen sie gleichwohl allein in die Produktion der Lebensmittel. Vgl. K. Marx u. F. Engels, Die deutsche Ideologie, Vorabdruck, 12-14, 16, 107 f. (MEW, Bd. 3, 20 f., 28-30). 
- sehen. ${ }^{39}$ Es entsteht die Frage, wie das Potenzial von Feuerbachs Sozialanthropologie zu mobilisieren wäre, ohne auf seine abstrakt-ideale Fassung der menschlichen Gattungsgeschichte zurückzufallen oder Marx' genetischen Struktur- und Prozessbegriff der Gesellschaft damit aufzugeben. Dazu im Folgenden einige Stichpunkte.

Hält man an Feuerbachs methodischen Grundgedanken fest, den Vergegenständlichungsund Vergesellschaftungsprozess genetisch von zwischenmenschlichen oder auch Beziehungen von Angesicht zu Angesicht, in der Elementarform vom Ich-Du-Bezug, aus zu erschließen, gelangt man zur Ausgangsebene eines unmittelbaren Umgangs der Individuen miteinander. ${ }^{40}$ Das Objekt wie die Natur erscheinen nach Feuerbach zunächst selbst in Form eines anderen Ich oder Du. ${ }^{41}$ Löwith hat bezogen auf diesen Ansatz später von einem Primat der Mitwelt gegenüber der Umwelt gesprochen. ${ }^{42}$ Der Umgang miteinander umfasst im weiten Sinn Bezüge gemeinsamen Erlebens, Fühlens, Leidens, Handelns, Sprechens und so weiter. Er ist nicht außer Raum und Zeit zu fassen, sondern innerhalb der geschichtlich geprägten alltagspraktischen Welt zu situieren, die eine Sphäre wiederkehrender, eingespielter Interaktionen und auch Interpassionen zwischen existierenden, konkreten Individuen ist. ${ }^{43}$ Insofern in die komplexe Alltagswelt immer schon die objektiven Vermittlungssphären, also Wirtschaft, Staat, Recht, Bildung, Religion und so weiter, hineinspielen, ist der unmittelbare Umgang miteinander jeweils einer bestimmten soziokulturellen Umwelt zuzurechnen. ${ }^{44}$ In dem unmittelbaren

39 Die Phrase von der Einheit von Ich und Du wäre bei Feuerbach nach Marx und Engels nicht möglich, ,wenn F. nicht an den Geschlechtsakt, den Gattungsakt, die Gemeinschaft von Ich \& Du $\varkappa a \tau$ '

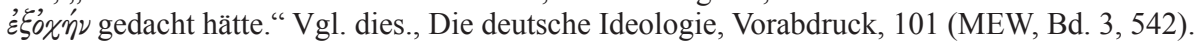

40 In seiner Theorie der Öffentlichkeit geht später in aufschließender Weise auch John Dewey methodisch und geschichtlich von Beziehungen von Angesicht zu Angesicht aus, die er der privaten Sphäre von lokalen Gemeinschaften zurechnet (vgl. ders., Die Öffentlichkeit und ihre Probleme, aus dem Amerikanischen von W.-D. Junghans, hg. v. H.-P. Krüger, Berlin 2001, bes. 48, 90; dazu ebenfalls S. Rost, Individualisierung, Vergesellschaftung und Vergemeinschaftung. John Deweys Öffentlichkeitsmodell und das Selbst bei George Herbert Mead, in: Deutsche Zeitschrift für Philosophie, 51 [2003] 5, 723737). Vergleichbar mit Feuerbach geht es Dewey darum, in der modernen Gesellschaft eine erneute (moralische) Gemeinschaft zu begründen - „die Suche nach Bedingungen, unter denen die Große Gesellschaft eine Große Gemeinschaft werden kann“ (ebd., 128). Im Unterschied zu dem Bruckberger Philosophen, der dieses Problem anhand der Religionsfrage lösen will, orientiert sich Dewey an der Sphäre der Öffentlichkeit und ihrer organisierten Form im demokratischen Staat.

41 Vgl. L. Feuerbach, Grundsätze der Philosophie der Zukunft, a. a. O., 316: „Der Begriff des Objekts ist ursprünglich gar nichts andres als der Begriff eines andern Ich - so faßt der Mensch in der Kindheit alle Dinge als freitätige, willkürliche Wesen auf -, daher ist der Begriff des Objekts überhaupt vermittelt durch den Begriff des Du, des gegenständlichen Ich.“

42 Vgl. K. Löwith, Das Individuum in der Rolle des Mitmenschen. 1928, in: ders., Sämtliche Schriften, Bd. 1, Stuttgart 1981, 27, 58 u. a.

43 Feuerbach hat den phänomenologischen Ausgangspunkt der Philosophie in der Lebenswelt bewusst ins Auge gefasst. Er schließt hier an Hegels phänomenologisches Konzept an, von der Unmittelbarkeit des sinnlichen Bewusstseins auszugehen, welcher Ausgangspunkt von ihm zur unmittelbaren Lebenswelt aufeinander bezogener Individuen transformiert wird. Feuerbachs kategorischer Imperativ lautet (Grundsätze der Philosophie der Zukunft, a. a. O., 334): „Wolle nicht Philosoph sein im Unterschied vom Menschen, sei nichts als ein denkender Mensch; [...] denke in der Existenz, in der Welt als ein Mitglied derselben, nicht im Vakuum der Abstraktion, als eine vereinzelte Monade, als ein absoluter Monarch, als ein teilnahmloser, außerweltlicher Gott." Die Philosophie hat nach Feuerbach entsprechend ,mit ihrer Antithese, mit der Nichtphilosophie, zu beginnen“(L. Feuerbach, Vorläufige Thesen zur Reformation der Philosophie, in: FGW, Bd. 9, 254).

44 Zur Bestimmung der Alltagswelt wie auch zu den folgenden Ausführungen vgl. G. Irrlitz, Über die Struktur philosophischer Theorien, in: Deutsche Zeitschrift für Philosophie, 44 (1996) 1, 3-30. 
Umgang miteinander manifestieren sich ursprünglich qualitative Bezugsmodi wie Erlebnisnähe, Intimität, Vertrautheit, Mitgefühl, Zuwendung, Liebe, Fürsorge, Ebenbürtigkeit oder Dialogizität, aber auch abgeleitete Formen wie Hass, Feindschaft, Gleichgültigkeit und anderes. ${ }^{45}$ Der von Husserl geprägte Alfred Schütz hat den unmittelbaren Umgang miteinander später der sozialen Umwelt zugeordnet: Das leibliche Du erscheint im Maximum seiner Symptomfülle, wobei das Erleben des Du nur als „Leben im inhaltserfüllten Wir“ möglich ist. ${ }^{46}$ Für Feuerbach liegt hier der genuine Entstehungsort und die Wirksphäre von individuellem Sein beziehungsweise Individualität. Der Einzelne gelangt nach ihm nur über den Bezug zu anderen zu einem originären Da- und Selbstsein. ${ }^{47}$ Der Ich-Du-Bezug steht in dieser Hinsicht nicht ursprünglich für austauschbare, symmetrische Rollen, ebenfalls nicht, wie Feuerbach vorgeworfen wurde, allein für geschlechtliche Beziehungen oder natürliche Eltern-Kind-Beziehungen; den Modus der Reziprozität und Zuwendung enthaltend, steht dieser Bezug ebenso für moralische Beziehungen zwischen konkreten Individuen. Feuerbach selbst hat sich in seinen Darstellungen vor allem am Phänomenfeld der christlichen Religion wie ihrer dechiffrierten Formen, an partnerschaftlicher, familiärer Liebe, Freundschaft, Moral, Bilder erzeugender Phantasie, ebenso an der kindlichen Entwicklung orientiert - Disziplinen wie Sozial-, Tiefen- und Entwicklungspsychologie oder soziologische Rollentheorien waren ihm noch unbekannt.

Beim Status eines unmittelbaren Umgangs miteinander ist nicht stehen zu bleiben; liegt die Eigentümlichkeit des Menschen für Feuerbach und Marx wie für ihre idealistischen Vorgänger doch eigens darin, das unmittelbare, partikulare Dasein zu transzendieren und zu einem soziokulturell vermittelten Gattungs- beziehungsweise Geistdasein aufzusteigen. Der Einzelne überschreitet notwendig den privaten familiären Kreis und geht in die Vermittlungssysteme von Bildung, Arbeit, Recht, Staat, Religion und so weiter ein. Auf der alltagspraktischen Ebene konstituiert sich in dieser Hinsicht ein eigener Vermittlungszusammenhang, in den die Strukturmomente der verschiedenen Betätigungsfelder einfließen, so die pädagogische Praxis, die kooperative Arbeitstätigkeit, gemeinsames politisches Handeln, religiöse Praktiken und so weiter. ${ }^{48}$ Die gegenseitigen Nahbeziehungen werden in der Folge durch ein spezifisch strukturiertes Gefüge gesellschaftlicher Verhältnisse überformt beziehungsweise auch substituiert, etwa durch unpersönliche Rechtsbeziehungen oder ein bloß sachhaft orientiertes Einander-Gebrauchen. Das unmittelbare Miteinandersprechen im Sinne von Verstehen

45 Zur qualitativen Bestimmung der Ich-Du-Beziehung vgl. auch die weiterführenden Darstellungen bei Karl Löwith und Martin Buber, die sich beide auf Feuerbach beziehen: K. Löwith, Das Individuum in der Rolle des Mitmenschen. 1928, a. a. O.; M. Buber, Ich und Du, 14. Aufl., Gütersloh 2005; und ders., Das dialogische Prinzip, 6., durchges. Aufl., Gerlingen 1992. Es wird sich ebenfalls auf Darstellungen bei Alfred Schütz und Max Scheler bezogen (siehe unten).

46 Vgl. A. Schütz, Der sinnhafte Aufbau der sozialen Welt. Eine Einleitung in die verstehende Soziologie, 5. Aufl., Frankfurt/M. 1991, 235, 231. Wie Scheler begreift Schütz die Ich-Du-Beziehung aus der Grundrelation des Wir (ebd., 230 f.).

47 Vgl. L. Feuerbach, Grundsätze der Philosophie der Zukunft, a. a. O., 317: „Nur in der Empfindung, nur in der Liebe hat ,Dieses ${ }^{`}$ - diese Person, dieses Ding -, d. h. das einzelne, absoluten Wert, ist das Endliche das Unendliche." Selbstsein und Individualität konstituieren sich Feuerbach zufolge so nur über das Miteinander der Individuen. Seine Anthropologie weicht damit deutlich von Stirners naturalistischem Ansatz ab, nach dem sich das Subjekt zur gegenständlichen und sozialen Welt primär im Modus von Nutzen und Gebrauch verhält. Vgl. M. Stirner, Der Einzige und sein Eigentum, mit einem Nachwort hg. v. A. Meyer, durchges. u. verb. Aufl., Stuttgart 1981, 331; zur Funktion der Welt als bloßes Material bei Stirner siehe ebd., 287, 330 u. a.

48 Die Aktivität der Individuen wird damit zugleich auf bestimmte Tätigkeitsformen festgeschrieben. Marx spricht in diesem Zusammenhang von einem „Sichfestsetzen der sozialen Thätigkeit“. Vgl. K. Marx u. F. Engels, Die deutsche Ideologie, Vorabdruck, 21 (MEW, Bd. 3, 33). 
und Missverstehen, Verständigen, Aufeinanderhören und so weiter geht in ein vom Einzelnen abgelöstes Alltagsvokabular über, das in medialer Form vorliegt. ${ }^{49}$

Plessner hat diese Seite der Alltäglichkeit eingehend geschildert: Das Individuum, um unangreifbar zu werden, legt sich gleichsam eine Rüstung an, mit der es den Kampfplatz der Öffentlichkeit betritt. Der Mensch maskiert sich und verzichtet zugleich auf sein Beachtet- und Geachtetwerden als Individualität. ${ }^{50}$ In seiner Fixierung der Lebenswelt auf die Arbeitswelt hat Marx hierbei vor allem die Subsumtion unter partikularisierende Arbeits- und antagonistische Klassenverhältnisse herausgestellt. Diese Sicht entsprang dem Erfahrungshorizont der sich konstituierenden Industriegesellschaft. Als soziale Grundmodi der Klassengesellschaften hat Marx in der Folge Konkurrenz, Ungleichheit, Ausbeutung und Herrschaft herausgehoben. Kommt der Arbeitswelt und dem Markt in diesen Modi nach wie vor eine wesentliche Stellung $\mathrm{zu}$ - selbst im Phänomen der Nicht-Arbeit -, so ist die komplexe Alltagswelt darauf doch nicht zu reduzieren, ebenso nicht auf reine Maskerade oder normiertes Rollendasein. ${ }^{51}$ Der Alltagsrealität ist unaufhebbar der Bezugsmodus reziproker Nahbeziehungen eingeschrieben, worin ein grundlegendes Reservoir an emotional-sinnlich fundierten, sozialen Bindungskräften liegt. Über den privaten, familiären Kreis hinaus sind gegenseitige, solidarische Beziehungen auch für die Welt der Arbeit, Erziehung oder Freizeit auszeichnend; ein vertrauensvoller, ebenbürtiger Umgang miteinander kann gleichfalls erst ein Resultat sein, das dem gesellschaftlichen Rollenspiel entspringt. ${ }^{52}$ Es ist zu fragen, inwieweit nicht nur Arbeit, sondern - neben ästhetischen, religiösen und anderen Äußerungsformen - eigens auch die sozialen Bindungskräfte den Vergesellschaftungsprozess über die Alltagsrealität hinaus bestimmen. Feuerbach hat einen solchen Zusammenhang herausgestellt, indem er die zwischenmenschlichen Beziehungen, insbesondere die Liebe, in der (christlichen) Religion zu gegenständlicher Gestalt kommen sieht, allerdings in undurchschauter Form. Durch einen allgemeinen Bewusstseinswandel in der Gesellschaft werden seiner Auffassung nach die religiösen Entfremdungsformen wieder in liebevolle Ich-Du-Bezüge zurückgenommen. Darin liegt eine stark idealisierende Lösung, hinter der bei dem Bruckberger Philosophen die pantheistische Eros-Tradition vorscheint, wonach aus höchster Erkenntnis notwendig Liebe entspringt.

Um auf die aufgeworfene Problematik zu antworten, ist mit Marx zur Ebene der objektiven Gesellschaftsstruktur und -entwicklung überzugehen. Dessen Leistung lag im nachhegelschen Philosophiediskurs darin, die subjektiven Ansätze Feuerbachs und der Junghegelianer zu überschreiten, wo sich vornehmlich auf die Subjektseite bezogen wurde, sei

49 Vgl. dazu bei Löwith den Abschnitt „Miteinandersein als Miteinander-Sprechen“, wo auf die Verselbständigung der Rede in Schrift eingegangen wird; ders., Das Individuum in der Rolle des Mitmenschen. 1928, a. a. O., 103-126.

50 H. Plessner, Grenzen der Gemeinschaft. Eine Kritik des sozialen Radikalismus [1924], in: ders., Gesammelte Schriften, hg. v. G. Dux, Bd. 5, Frankfurt/M. 2003, 82.

51 Vgl. dagegen E. Goffman, Wir alle spielen Theater. Die Selbstdarstellung im Alltag, München 1999. Zur kritischen Sichtung der Alltagswelt vgl. etwa R. Barthes, Mythen des Alltags, Frankfurt/M. 1964; H. Lefebvre, Kritik des Alltagslebens. Grundrisse einer Soziologie der Alltäglichkeit. Mit einem Vorwort zur deutschen Ausgabe, aus dem Französischen v. B. Kroeber u. K. Held, Frankfurt/M. 1987.

52 In seiner von Feuerbach ausgehenden Strukturanalyse des Miteinanderseins hat Löwith die Beziehung von Ich und $\mathrm{Du}$, wie sie eine freie Anerkennung der Ebenbürtigkeit und Selbständigkeit des einen durch den anderen einschließt, überhaupt nur als einen vermittelten, das alltägliche EinanderGebrauchen überschreitenden Bezug aufgefasst, wozu er neben Feuerbachs Anthropologie ebenfalls auf Kants Ethik zurückgreift; vgl. ders., Das Individuum in der Rolle des Mitmenschen. 1928, a. a. O., $153 \mathrm{ff}$. 
es in Form von Liebe, reflektierter Intellektualität, egoistischem Interesse oder willentlicher Praxis. Marx ging in kritischer Absetzung von diesen Ansätzen zur Betrachtungsebene der geschichtlich realisierten gesellschaftlichen Vermittlungen über, wozu er eine spezifische Schichtung der soziokulturellen Verhältnisse zu Grunde legte. Eine zusammenfassende Darlegung seines gesellschaftstheoretischen Ansatzes findet sich etwa im Vorwort zur Kritik der Politischen Ökonomie. Als Leitfaden seines Ansatzes stellt er dort eine Aufschichtung der Gesellschaft in die Basissphäre der Produktionsverhältnisse heraus, worüber sich die Überbausphäre der juristischen und politischen Verhältnisse sowie die gesellschaftlichen Bewusstseinsformen erheben. ${ }^{53}$ Die Arbeit, wie sie den Stoffwechsel des Menschen mit der Natur vermittelt, bildet die fundamentale Strukturierungsform des Vergegenständlichungs- und Vergesellschaftungsprozesses. Marx drückt diesen Ansatz auch im Primat des materiellen (ökonomischen) Seins aus, wogegen die ideologischen Formen (juristische, politische, religiöse, künstlerische, philosophische) einen nachgeordneten Status besitzen ${ }^{54}$ - in Absehung gleichwohl vom Umstand, dass auch der ökonomische Lebensprozess auf das Bewusstsein bezogen ist. Die Eigenstruktur und -dynamik der so genannten ideologischen Formen tritt damit in den Hintergrund. ${ }^{55}$

Hält man an Marx' phänomenologischem Vergegenständlichungskonzept fest, ohne diese duale Hierarchie der Tätigkeits- und Objektivierungsformen zu teilen, ist der objektive Vermittlungsprozess der Menschen in weitergehender Form zu fassen. Greift man in diesem Zusammenhang auf Feuerbachs sozialanthropologische Basis zurück, ist der geschichtliche Objektivierungsprozess ebenfalls im Hinblick auf die Vergegenständlichung der gegenseitigen Nahbeziehungen zu reflektieren. Die Reproduktion des materiellen Lebens ist in dieser Hinsicht nicht mit der Reproduktion des sozialen Zusammenhangs gleichzusetzen, wie dieser aus dem unmittelbaren Umgang miteinander hervorgeht. Die soziale Integration bildet vielmehr einen eigenständigen geschichtlich realisierten Vermittlungsstrang, der sich um die objektiven moralischen, rechtlichen und politischen Verhältnisse zentriert und in der Frühphase der menschlichen Evolution mit dem System der verwandtschaftlichen Beziehungen eingesetzt hat. ${ }^{56}$ Infolge der weiteren gesellschaftlichen Entwicklung in den frühen Hochkulturen (Produktivkraftentwicklung, Teilung der Arbeit in Stadt und Land, soziale Differenzierung, Klassenbildung und so weiter) ist anstelle der verwandtschaftlichen Beziehungen

53 K. Marx, Zur Kritik der politischen Ökonomie. Erstes Heft, in: $\mathrm{MEGA}^{2}$, Bd. II/2, 100.

54 Ebd., $100 \mathrm{f}$.

55 In seiner Studie zur Ideologie hat Terry Eagleton in diesem Zusammenhang auf ein antithetisches Moment im Marxschen Ansatz gewiesen, mit dem der materielle Lebensprozess und die Ideen beziehungsweise das Bewusstsein (Ideologie) unzulänglich einander gegenübergestellt werden. Eagleton sieht in dieser Schematik eine Abstraktion, insofern ,es keinen ,wirklichen Lebensprozeß ohne Bewußtsein“" gibt. Die abstrakte Fassung bei Marx liegt für ihn in einer bloßen Umkehr des idealistischen Ansatzes der Junghegelianer, bei denen im Unterschied zu Marx die Ideen den materiellen Lebensprozess bestimmen (vgl. ders., Ideologie. Eine Einführung, aus dem Engl. v. A. Tippner, Stuttgart 2000, 88 f.). Unabhängig von diesen methodischen Grundprämissen reflektiert Marx, vor allen in seinen zeitgeschichtlichen Studien, durchaus eigene rechtliche, politische, moralische und so weiter Zusammenhänge. Diese Seite wird hier ausgeblendet. Zu Marx' Auffassungen zu Moral und Ethik vgl. zum Beispiel die beiden Aufsätze von Andreas Wildt in der Deutschen Zeitschrift für Philosophie von 2002 (Heft 3 und Heft 5).

56 Ebenfalls Marx hat die Familie als ,,im Anfange das einzige soziale Verhältniß“ bestimmt; K. Marx u. F. Engels, Die deutsche Ideologie, Vorabdruck, 14 (MEW, Bd. 3, 29); vgl. auch Marx’ Ausführungen zum naturwüchsigen Gemeinwesen in seinen ökonomischen Schriften wie seine ethnologischen Exzerpte, Letztere in: The ethnological notebooks of Karl Marx (Studies of Morgan, Phear, Maine, Lubbock), hg. v. L. Krader, Assen 1972. 
dann die staatliche Organisation beziehungsweise politische Herrschaft getreten. ${ }^{57}$ Über den geltenden Moralzusammenhang hinaus fungierte der Staat als eine eigene organisierte Wirkund Entscheidungseinheit. Das Recht kann man in bestimmter Hinsicht als eine Vermittlung zwischen Politik und Ethik auffassen. ${ }^{58}$ In die politische und rechtliche Vermittlungssphäre fließen ökonomische Interessen und Prozesse ein, ebenso geht es darin, wie Hasso Hofmann bezogen auf die moderne Rechtssprechung festgehalten hat, um eine „kontrollierte Überführung von sozialem Ethos, von objektiver gesellschaftlicher Sittlichkeit in die Inhalte von Verfassung und Recht". ${ }^{59}$ Diesen Sphären kommt damit eine spezifische, individuelle und kollektive Interessen zu gesamtgesellschaftlicher Regulierung und Macht vermittelnde Funktion zu.

Zur systematischen Darlegung der politischen und rechtlichen Sphäre kann sich zweifellos weder auf den im Ich-Du-Bezug verbleibenden Ansatz Feuerbachs noch auf Marx politische Auffassungen allein gestützt werden. Marx begreift den Staat im Wesen als Instrument der ökonomisch herrschenden Klasse, der für die beherrschten Klassen nur eine illusorische Allgemeinheit bildet. ${ }^{60}$ Eine eigenständige Sozialisierungsleistung außerhalb der ökonomischen Reproduktion kommt der politischen und auch rechtlichen Sphäre bei ihm nicht zu. Marx hat in dieser Hinsicht nicht den Aspekt gesehen, dass die politische und rechtliche Vermittlungssphäre Tendenzen bergen, die im Hinblick auf Regulierungs-, Ausgleichs- und Verteilungsfunktionen dem ökonomischen Verwertungsprozess auch entgegenwirken können. Solche entgegensteuernden Kräfte finden sich in der geschichtlich realisierten Institution des sozialen Rechtsstaats, sie sind aber auch für andere soziokulturelle Sphären auszeichnend. In der gegenwärtigen Phase des global agierenden Kapitalismus zeigt sich gleichwohl die Tendenz, dass unter Vorherrschaft einer ,monetären Integration“ ${ }^{\text {"61 }}$ bisher erreichte sozialstaatliche Leistungen wieder aufgehoben werden und die Internationalisierung rechtlicher und

57 Vgl. dazu die im Rahmen der Rekonstruktionsversuche des historischen Materialismus geführte Diskussion um die Entstehung von Klassengesellschaften: Seminar: Entstehung von Klassengesellschaften, hg. v. K. Eder, Frankfurt/M. 1973; K. Eder, Entstehung staatlich organisierter Gesellschaften, Frankfurt/M. 1976.

58 E.-W. Böckenförde hat das Recht in seiner Funktion einer normativen Abstützung und Sicherung der vorhandenen sozialen Lebenswirklichkeit als eine "Vermittlung zwischen Politik und Ethik“ umschrieben, wobei er Ethik als den Gesamtbereich des in der Gesellschaft wirklichen Sittlichen versteht, das seinerseits aus verschiedenen Quellen (Mythos, Religion, Freiheitsethik) geformt sein kann. Das Recht entsteht nach Böckenförde aus der Entzweiung von Politik und (präsenter) Ethik (vgl. ders., Staat, Gesellschaft, Freiheit. Studien zur Staatstheorie und zum Verfassungsrecht, Frankfurt/M. 1976, 26, 28). Das Recht, lässt sich ergänzen, kann ebenfalls als Vermittlungsform zwischen Politik und der durch geschichtlich realisierte Produktions-, Austausch- und Eigentumsverhältnisse geprägten Wirtschaft angesehen werden.

59 H. Hofmann, Einführung in die Rechts- und Staatsphilosophie, 3., durchges. u. korr. Aufl., Darmstadt 2006, 29.

60 Vgl. K. Marx u. F. Engels, Die deutsche Ideologie, Vorabdruck, 94 f. (MEW, Bd. 3, 62). Bezogen auf die Klassengesellschaften legt Marx einen instrumentellen Politikbegriff in der Hobbesschen Tradition zu Grunde, den er ideologiekritisch und klassentheoretisch fundiert.

61 Vgl. diesen Begriff bei Pierre Bourdieu, so in: ders., Die fortschrittlichen Kräfte, in: ders. u. a., Perspektiven des Protests. Initiativen für einen europäischen Wohlfahrtsstaat, Hamburg 1997, 12. Um der Vorherrschaft der ökonomisch-monetären Integration in Europa entgegenzutreten, spricht sich Bourdieu für eine Internationalisierung des Sozialrechts und Wohlfahrtstaates aus. Er plädiert für einen europäischen Sozialstaat, in dem ein gemeinsames Sozialrecht, eine Festsetzung von Mindestlöhnen, eine gemeinsame soziale und ökologische Investitionspolitik und anderes angestrebt wird (ebd., 23 f.). 
politischer Formen auf eine machtpolitische, verwaltungstechnische Weise erfolgt. Wie in der Wirtschaft kommt es ebenfalls in der politischen und rechtlichen Sphäre zu spezifischen Verselbständigungs- und Versachlichungsprozessen.

Kommt man am Ende auf die Emanzipationsthematik zurück und fragt, inwieweit sich die Menschheit auf eine Gesellschaft zubewegt, in der, wie Marx antizipiert hat, der wirkliche individuelle Mensch in seiner konkreten Lebenswelt zu einem erfüllten Leben in Gemeinschaft mit anderen gelangt ${ }^{62}$, oder im Gegenzuge dazu unter verselbständigte Sachwelten subsumiert wie atomisiert wird, ist darauf nur im Horizont aller Äußerungs- und Objektivierungssphären wie ihrer geschichtlichen Ausdifferenzierung zu antworten; bilden die Menschen doch in allen Sphären, der wirtschaftlichen, politischen, rechtlichen, moralischen, religiösen und so weiter, ihre individuellen, gemeinschaftlichen und kulturellen Potenziale aus. Die Emanzipationsfrage ist in dieser Hinsicht nicht auf einzelne Realisierungssphären wie die ökonomische oder moralische zu beschränken. Die verschiedenen soziokulturellen Sphären enthalten in ihrer Eigengesetzlichkeit potenziell Strukturierungsprinzipien, die den destruktiven Folgen der Kapitalverwertung entgegenlaufen können. Auf der objektiven Vermittlungsebene kommen entgegenlaufende Tendenzen dem rechtlichen und politischen Prozess, in idealer Form auch der kritischen Öffentlichkeit, Kunst oder dem philosophischen Diskurs zu. Marx hat dem ökonomischen Prozess selbst das Moment einer immanenten Selbstüberschreitung der Kapitalproduktion zuerkannt. ${ }^{63}$ Fragt man danach, wie die potenziellen Sozialisierungsformen de facto in den realen gesellschaftlichen Lebensprozess eingehen, ist von der objektiven Vermittlungsebene der soziokulturellen Systeme wieder zur alltagspraktischen Welt als dem eigentlichen Zielort „menschlicher Emancipation“ zurückzukehren; beruhen die institutionalisierten Systeme der Wirtschaft und des Staates doch auf beharrenden Steuerungsmechanismen beziehungsweise unterliegen selbst latent Verselbständigungsprozessen. Es sind die der praktischen Lebenswelt entspringenden sozialen Bewegungen, die auf reelle gesellschaftliche Veränderungen drängen. Darunter fallen Gewerkschafts-, Ökologie-, Frauen-, Friedens- und Bürgerbewegungen ebenso wie die Aktivitäten der Globalisierungsgegner. Diese Bewegungen sind nicht auf ein exklusives Subjekt festzuschreiben, etwa auf die Arbeiterklasse oder die so genannte „Dritte Welt“. Die sozialen Bewegungen werden von materiellen Interessen und Existenznot geleitet, man denke etwa an die neuen Bürgerkriege; ein wesentlicher Anschub geht darüber hinaus von der gelebten Moral und den solidarischen Bindungskräften in der Alltagswelt aus, vermittelt ebenfalls von intellektueller Einsicht. Werden in der Lebenswelt auftretende Bezugsmodi wie Reziprozität, Fürsorge oder Daseinssicherheit dauerhaft verletzt, so kann Enttäuschung über

62 Vgl. K. Marx, Zur Judenfrage, in: MEGA², Bd. I/2, 162 f.

63 Durch Produktivkraftentwicklung, insbesondere auf Basis der Wissenschaft, hört nach Marx die Arbeitzeit perspektivisch auf, allgemeines Maß des Reichtums zu sein, wie der Tauschwert nicht mehr als Maß des Gebrauchwerts auftritt. Die auf dem Tauschwert beruhende Produktion bricht nach ihm in der Folge zusammen. Die Reduktion der notwendigen Arbeitszeit auf ein Minimum findet nicht statt, um Surplusarbeit zu setzen, sondern führt Marx zufolge zu einer freien Entwicklung der Individualität (vgl. ders., Grundrisse der Kritik der politischen Ökonomie. Zweiter Teil, in: $\mathrm{MEGA}^{2}$, Bd. II/1, 581 f.). Die gegenwärtige Entwicklung zeigt allerdings, dass die intellektuelle Tätigkeit selbst unter die Tauschwertproduktion fällt, wie die Mehrheit der von Lohnarbeit Befreiten nicht allein von Arbeit, sondern zugleich von grundlegenden gesellschaftlichen Gütern ausgeschlossen wird. Die ökonomische Vergesellschaftung führt insofern nicht automatisch zu einer Überschreitung des Kapitalverhältnisses. Eine äußere Schranke kann man vielmehr in der in ihren Ressourcen begrenzten Natur sehen, die gegenwärtig als stärkster Widerpart bezogen auf die Kapitalverwertung auftritt. 
das „Dasein eines Unwertverhaltes“ oder den „Mangel eines positiven Wertverhaltes““64, um mit Max Scheler zu sprechen, perspektivisch in soziale Widerstandsbewegungen umschlagen. Es besteht zugleich die Möglichkeit, dass daraus soziale Hassbewegungen entstehen, die wie der Terror eine destruktive Wirkung auf die Gesellschaft besitzen können. ${ }^{65}$ Auch in dieser Hinsicht sind die sozialen Bewegungen nicht mit einem kognitiven Lernprozess zu identifizieren. ${ }^{66}$ Eine weiterführende Frage ist, inwieweit die sozialen Gegenbewegungen tatsächlich qualitative Strukturveränderungen auf der institutionellen Vermittlungsebene bewirken, was nur auf der empirischen Ebene konstatierbar ist. Die Emanzipationsfrage ist auf diese Weise nicht losgelöst von der objektiven Strukturanalyse der Gesellschaft zu behandeln, und zwar im Spannungsfeld von objektiven gesellschaftlichen Gegebenheiten und sozialer Aktivität. Die geschichtliche Perspektive liegt damit in einem offenen Prozess, der einen entwicklungslogischen Automatismus, sei es ökonomischer oder logischer Art, ausschließt.

Dr. Christine Weckwerth, Berlin-Brandenburgische Akademie der Wissenschaften, Jägerstraße 22/23, 10117 Berlin

64 M. Scheler, Ordo Amoris, in: ders., Gesammelte Werke, Bd. 10: Schriften aus dem Nachlaß, Bd. 1, 3., durchges. Aufl., hg. v. M. Frings, Bonn 1986, 369.

65 Vgl. dazu H.-P. Krüger, Hassbewegungen. Im Anschluss an Max Schelers sinngemäße Grammatik des Gefühlslebens, in: Deutsche Zeitschrift für Philosophie, 54 (2006) 6, 867-883.

66 Die Auffassung, soziale Bewegungen als Lernvorgänge zu begreifen, vertritt dagegen Jürgen Habermas. Über sie werden nach Habermas (Zur Rekonstruktion des Historischen Materialismus, a. a. O., 40) „latent bereitstehende Rationalitätsstrukturen in gesellschaftliche Praxis umgesetzt", die schließlich zu institutioneller Verkörperung gelangen. 